\title{
Analysis of the Pimelea toxin simplexin for the development of a cattle microbial probiotic
}

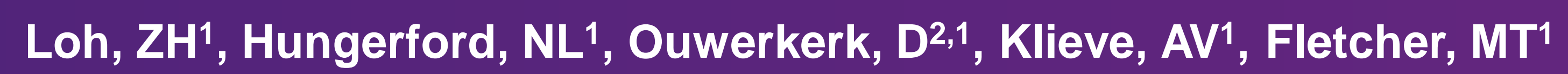

${ }^{1}$ Queensland Alliance for Agriculture and Food Innovation (QAAFI), The University of Queensland, Health and Food Sciences Precinct, Coopers Plains, QLD 4108, Australia

${ }^{2}$ Agri-Science Queensland, Department of Agriculture and Fisheries (QDAF), Ecosciences Precinct, Dutton Park, QLD 4102, Australia

\section{Introduction}

Pimelea poisoning of cattle (Fig. 1) is a uniquely Australia poisoning caused by the toxin simplexin (Fig. 2), found in native Pimelea plants [1]

- To date, there is no effective treatment for Pimelea poisoning.

- Cattle fed a diet containing increasing low doses of simplexin displayed reduced poisoning signs over time [2], suggesting adaption of rumen microorganisms to detoxify simplexin.

- This project aims to develop a protective microbial probiotic derived from the rumen fluid of field-exposed animals that is capable of detoxifying simplexin.
Figure 1: Steer affected by Pimelea poisoning



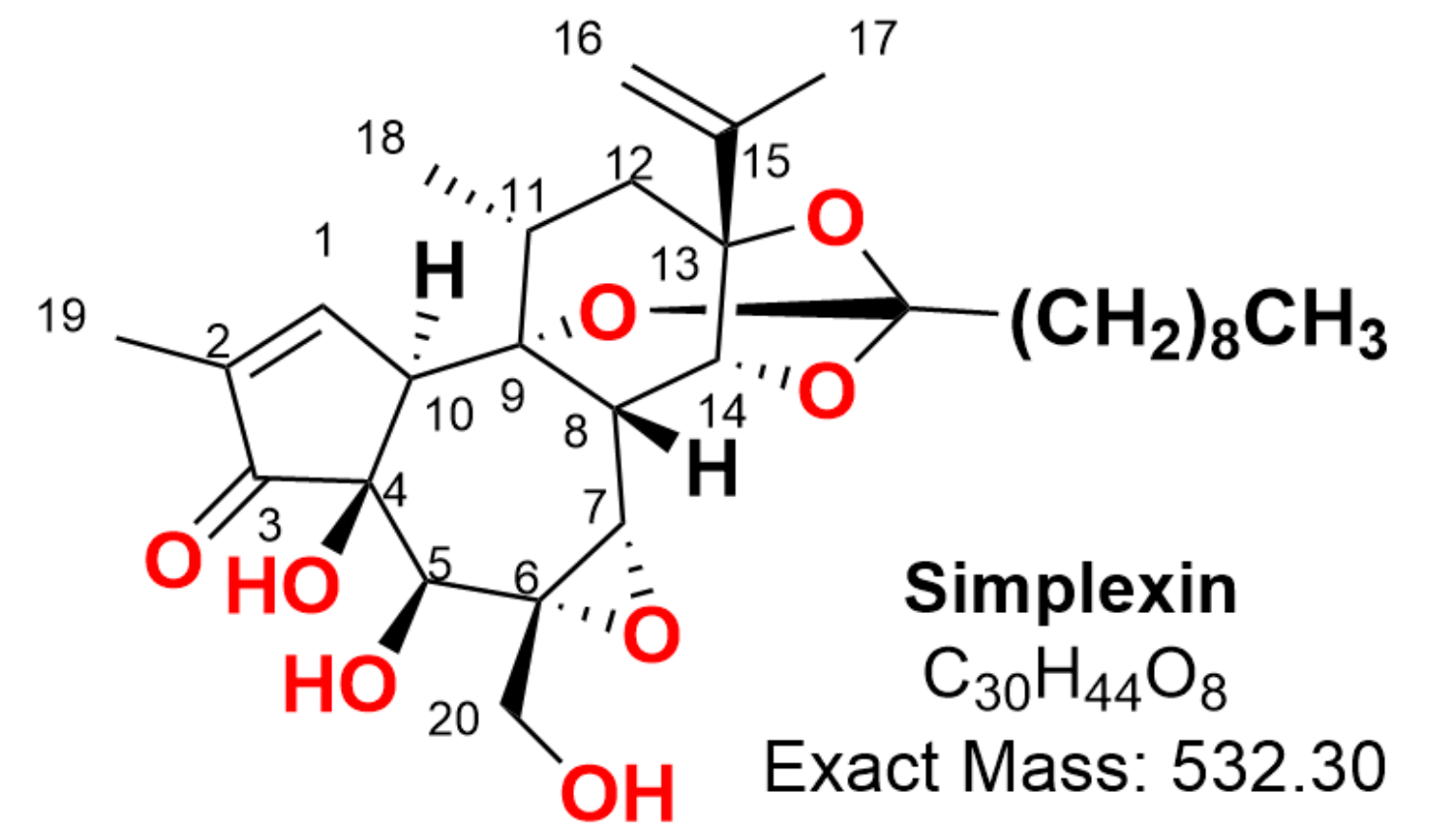

Figure 2: Simplexin chemical structure

\section{Results \& Discussion}

- Quantification of simplexin in both in-vitro studies showed decreased levels of simplexin.

- Acid hydrolysis of simplexin resulted in the identification of six possible hydrolysed simplexin products based on predicted molecular formulae (Fig. 4).

- Possible hydrolysed simplexin products also shared several fragmentation ions with simplexin.

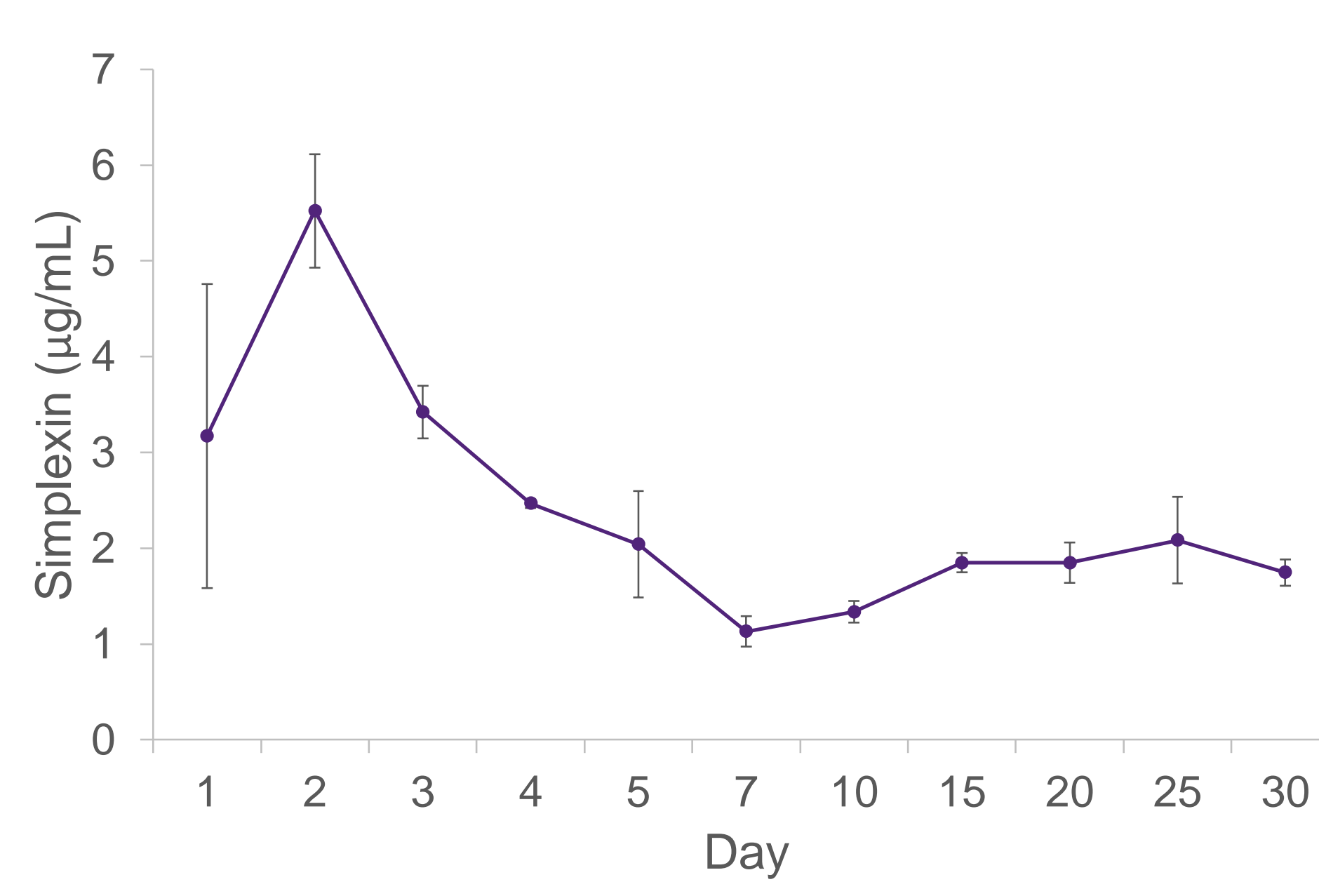

Figure 3: Simplexin levels in in-vitro rumen fermentation study

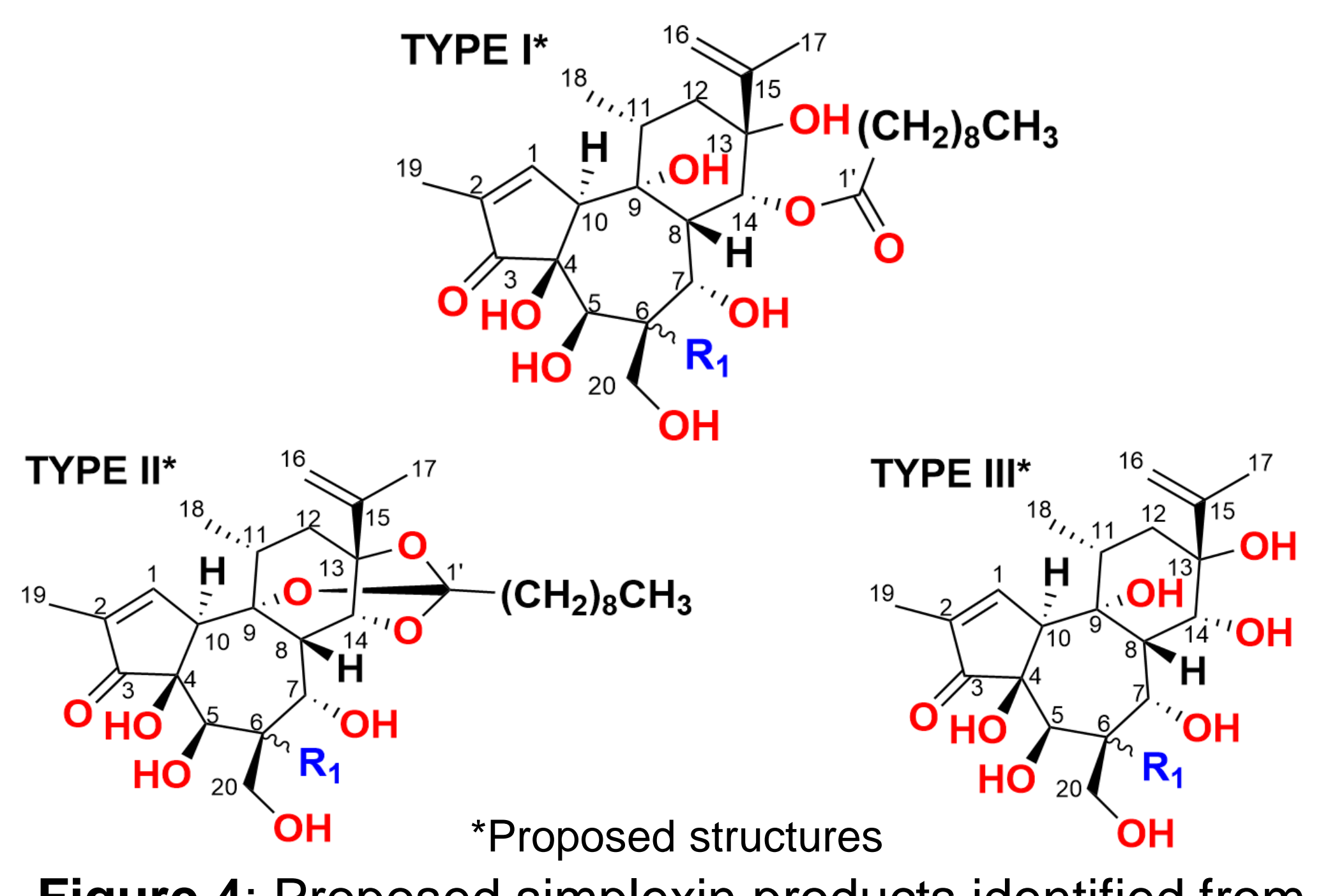

Figure 4: Proposed simplexin products identified from hydrolysis with aq. $\mathrm{HCl}\left(\mathrm{R}_{1}=\mathrm{Cl}\right)$ and $\mathrm{H}_{2} \mathrm{SO}_{4}\left(\mathrm{R}_{1}=\mathrm{OH}\right)$

\section{Methods \& Materials}

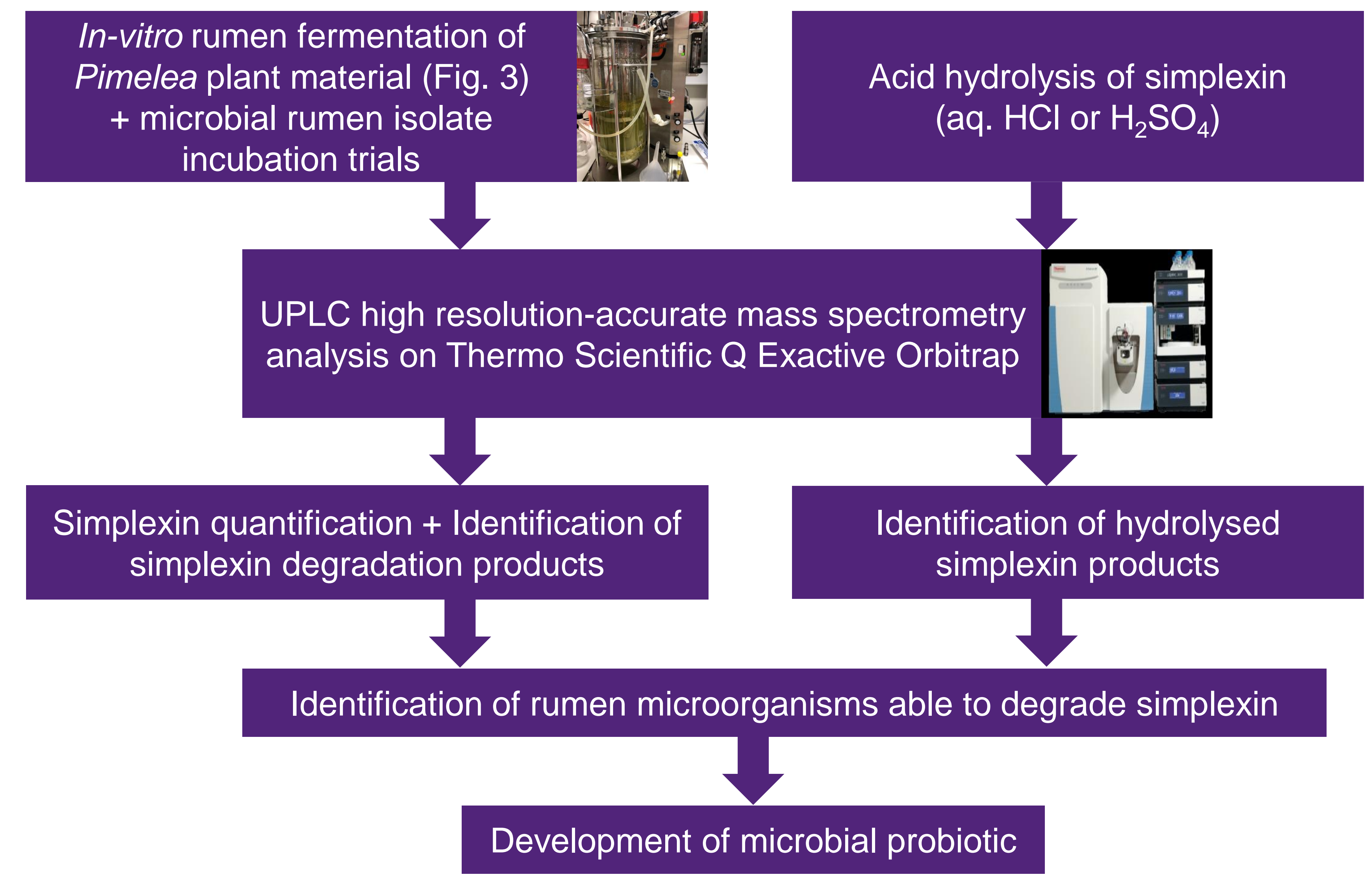

\section{Conclusions}

- UPLC-MS/MS analysis enabled simplexin quantification at low concentrations $(\mathrm{ng} / \mathrm{mL})$ - Simplexin decrease in in-vitro studies suggestive of potential simplexin degradation by rumen microorganisms.

- Possible simplexin hydrolysed products can be identified using the analytical method.

\section{Future studies}

- Identification and characterisation of simplexin degradation products in both in-vitro fermentations and bacteria isolate incubation studies.

- Identified rumen isolates capable of degrading simplexin will be further investigated.

References

[1] P. Freeman, E. Ritchie, W. Taylor, Australian Journal of Chemistry, 1979, 32, 2495-2506. [2] MT. Fletcher, S. Chow, SM. Ossedryver, Journal of Agriculture and Food Chemistry, 2014, 62, 7402-7406.

\section{uq.edu.au}

Queensland Alliance for Agriculture and Food Innovation

me :U: THE UNIVERSITY $\operatorname{minf}_{\substack{\text { OF QUEENSLAND } \\ \text { U S T R A L I A }}}$ 